\title{
Elfte Versammlung der italienischen Gesellschaft für Dermatologie und Syphilographie.
}

In den Sitzungen, die vom 20.-23. Dezember 1909 in Rom statt hatten, wurden folgende Vorträge gehailen:

Barduzzi und Simonelli. Hereditäre Syphilis.

Ayala. Hautinfiltration des inneren Blattes des Präputiums, die eine syphilitische Initialsklerose vortäuschte, als Komplikation eines gonorrhoischen Prozesses.

Campana. Sporotrichosis.

Carnevali. Über die sogen. parasyphilitischen Manifestationen.

Chirivino. Die Farbreaktion Schürmanns zur Diagnose der Syphilis.

Cipolla. Klinischer Beitrag zur Wassermannschen Reaktion.

Mibelli und Philippson. Dermatologische Nomenklatur.

Das Thema enthielt zahlreiche wichtige Vorschläge und soll in den kommenden Sitzungen der Gesellschaft weiter erörtert werden.

Pini. Betrachtungen über die Serodiagnose Wassermanns.

Gavini. Serodiagnose Wassermanns.

Lombardo, C. und Tognoli, E. Über die Passage des Quecksilbers von der Mutter zum Fötus.

Mannino. Zwei neue Fälle von Abortierung der Syphilis mittels intensiver und frühzeitiger $\mathrm{Hg}$-Behandlung.

Mariani. Syphilis und Ehe. auf Tiere.

Truffi. Untersuchungen über die Übertragbarkeit der Syphilis

Minassian. Modifikation zu den Methoden der Untersuchung auf Spirochaeta pallida. Schnelle Imprägnation der syphilitischen Gewebe mit Argent. nitricum. Es werden zwei Lösungen gebraucht: 1. Argent nitric. 1:5, Formol 5॰0, Alkohol 50 0. 2. Acid. pyrogall. 3·5, Formol 10.0, Alcohol absol. 100.0. Die Stücke bleiben 24 St. in Lösung 1 bei $32-35^{\circ}$, dann ebenfalls 24 St. bei derselben Temperatur in Lösung 2.

Dann Alkohol, Xylol.

Sabella. Das kalte Bad (in Regenform) bei rezenter Syphilis.

Terzaghi. Unsere Resulsate bei der Untersuchung auf Spirochaeta pallida.

Areh. f. Dermat, a. Syph. Bd. UIV. 

infantilis.

Terzaghi. Die Wirkungen der Röntgenstrahlen bei Pruritus

Ossola. Weitere Untersuchungen über die Übertragbarkeit der Syphlis auf Kaninchen.

Ossola. Syphilis des Hodens beim Kaninchen.

Dalla Favera. Weitere Beobachtungen über den Gebrauch des "Arsacetin" bei der Behandlung der Syphilis.

Stancanelli. Über den Wert des Arsacetin von Ehrlich bei der Behandlung der konstitutionellen Syphilis.

Fioc co. Neue Untersuchungen über den Boubas (Framboesia tropica) Brasiliens.

Verrotti. Histologische und balkteriologische Untersuchungen bei drei Fällen von brasilianischem Boubas (mit Demonstration von Zeichnungen, mikroskopischen Präparaten, Photographien und Moulagen).

Lom bardo, C. Ampullen für unlösliche Quecksilberpräparate.

Mazza. Kulturversuche im Reagensglase der Spirochaeta pallida.

Radaeli. Bakteriologische Untersuchungen bei Pemphigus (mit Demonstration von Kulturen und mikroskopischen Präparaten).

Simonelli. Beitrag zum Studium der Alterationen des Knochenmarks bei Pemphigus.

Stancanelli. Dermatitis pemphigoïdes diffusa mit akutem und gutartigem Verlauf durch Autointoxikation alimentären Ursprungs.

Fi occo. Die Trichophytien in Venedig.

Majocchi. Weitere klinische Beobachtungen und experimentelle Untersuchungen über das Granuloma trichophyticum.

Radaeli. Eigenartiger Fall von Keratoma palmare et plantare.

Vignolo-Lutati. Über einen Fall von periodischer Alopekie (mit Demonstration von Photographien und Mikrophotographien).

Sim on elli. Intravenöse Inokulationen von gonorrhoischem Eiter bei Kaninchen.

Dalla Favera. Erythema elevatum diutinum und Granuloma anulare.

Ercoli. Weitere Untersuchungen über einen Fall von Urticaria pigmentosa beim Erwachsenen (mit Demonstration mikroskopischer Präpárate).

Eccoli. Über einen Fall von generalisierter Sklerodermie mit Leberzirrhose. (Klinischer Beitrag zum Studium der Läsionen innerer Organe bei Sklerodermie.)

Pelagatti. Über eine noch nicht beschriebene Varietät von Mikrosporum.

Majocchi, D. Über die Dermato-myiasis muscosa. carnaria.

Mibelli, V. Zwei Fälle von Myiasis cutanea durch Sarcophaga

Verrotti. Über einen eigenartigen Fall von Lupus erythematodes diffusus am ganzen Kopf und an den Händen. (Klinischer, histologischer und pathogenetischer Beitrag.) 
Verrotti. Über einen Fall von Periphlebitis der unteren 1. Extremität, die eine zirkumskripte Sklerodermie vortäuschte.

Ciarrocehi. Über eine Varietät von Hantgangrän bei Wickelkindern durch chemische Ursachen.

Pecori. Über Dermatosen durch die Verwendung von Paraphenylendiamin zu kosmetischen Tinkturen.

Truffi. Ein Fall von Lepra in Savona.

Serra. Beitrag zum Studium des Bazillus Hansen.

Rivalta. Bedeutung des Studiums der Simulation bei Dermatosen.

Sberna. Krätze durch Korn.

Define. Lichen planus obtusus. (Klinische, histologische und bakterioskopiscbe Beobachtungen mit experimentellen Untersuchungen.)

Define. Ausgedehntes ulzero-serpiginöses dorsolumbales Syphiloderm infolge Syphilis ignorata.

Lanzi. Einige mit Atoxyl geheilte Fälle von Psoriasis.

La Mensa. Lichen scrophulosorum spinulosus.

La Mensa. Lichen planus mit ausschließlich annulärer Form, mit atypischem Auftreten bei einem Psoriatiker.

A yala. Über einen Fall von Prurigo. Dubreuilh (bei Lymphdrüsenerkrankung).

Carruccio. Spezifische Meningo-myelitis.

Vignolo-Lutati. Über zwei Fälle von Recklinghauseascher Krankheit (mit Demonstration von Photographien).

Segré, H. Ein Fall von Bromoderma tuberosum nach Gebrauch von Bromural.

Garibaldi. Günstige Resultate bei der Behandlung der chronischen Urethritis mit einer Kanüle zu doppeltem Gebrauch.

Garibaldi. Inokulationsversuche mit leprösem Metrial in den Hoden eines Hammels.

Galimberti. Die chemischen Strahlen des Spektrums bei der Germination nnd den Dermopathien.

Maz za. Polymorphes bnllöses Erythem mit Ausgang in Ulzeration.

Ercoli. Beschreibung zweier atypischer Fälle von Psoriasis (mit Demonstration von Photographien).

Ercoli. Atrophie der Glans infölge neoplastischer und chroniseh entzündlicher Prozesse des Präputiums (mit Demonstation anatomischer Stücke und mikroskopischer Präparate). J. U1lman n (Rom). 